Review Article

\title{
CME; Conceptualising Research in Medicine
}

\author{
Badrinarayan Mishra
}

Professor, Community Medicine, R.D. Gardi Medical College, Ujjain, Madhya Pradesh, India.

DOI: https://doi.org/10.24321/2394.6539.201909

\section{I $\quad \mathbf{N} \quad \mathbf{F}$}

E-mail Id:

badrinmishra@gmail.com

Orcid Id:

https://orcid.org/0000-0001-6956-0469

How to cite this article:

Mishra B. CME; Conceptualising Research in Medicine. J Adv Res Med Sci Tech 2019; 6(3\&4):

25-30.

Date of Submission: 2020-03-10

Date of Acceptance: 2020-03-20

\section{$\begin{array}{llllllll}\mathbf{A} & \mathbf{B} & \mathbf{S} & \mathbf{T} & \mathbf{R} & \mathbf{A} & \mathbf{C} & \mathbf{T}\end{array}$}

Understanding the basic principles of research for medical professionals seems pretty intricate. This CME is aimed at presenting a thematic conceptual framework for them. The seemingly uncomprehending terms of ontology and epistemology are explained and their role in building up research concept elaborated. The methodology and methods in pursuit of the relevant ontological foundation and epistemological basis are amalgamated so that any physician can understand and explore the researching documents and explore the avenue on their own accord.

Keywords: Research, Ontology, Epistemology, Methodology, Methods

\section{Introduction}

Research is 'robust search'; a stringent method by which an 'idea' or 'evidence' or 'truth' is searched and substantiated or proved. Traditionally 'researcher' or 'truth seekers' are known as philosophers. In modern time the concept of philosophy (philosopha; Greek; 'for love of wisdom') is no more confined to spirituality alone. It has diversified and is having wider applicability encompassing all life spheres. ${ }^{1-3}$

The search for truth or genuineness has become empirical. To make truth verifiable we need to do research. This process involves meticulous approach.

As a first step, the researcher has to identify the existing knowledge and propose a hypothesis. This can be achieved via the ontological concept of relativism, EMIC epistemology, qualitative methodology and qualitative approaches or descriptive methods stemming from realistic ontology, ETIC epistemology and quantitative methodology. ${ }^{4-7}$

The proposed hypothesis then can be tested through the rigour of analytical research is based on realistic ontology, ETIC epistemology and quantitative methodology and methods. ${ }^{8-11}$

Let us look into these somewhat bombastic terminologies from a close quarter.

\section{Research Paradigm}

Doing a thing successfully requires a lot of planning. The planning process in the conduction of research is known as its paradigm. As we have seen research is 'finding truth' and this can be achieved in different ways. If we perceive truth as an 'absolute 'that can be measured and substantiated then we are adopting a path of realism and the consequent processes of realistic ontology, ETIC epistemology and quantitative methodology. ${ }^{12-14}$ On the other hand, if we believe the truth is a subjective phenomenon and try to measure it as it is perceived by the population; then we are believed to pursue a relative ontological approach that leads to EMIC epistemology and qualitative methodology. ${ }^{12-15}$

\section{Ontology}

Ontology is the first step in the research paradigm which shall lead us in the right direction. Here we try to deals with existing belief. If a researcher believes in certain things or concepts which he or she can measure as a quantifiable item then the ontology followed is termed as realistic or objective or quantitative. ${ }^{16-18}$ But on the other hands if the researcher is exploring something for the first time and doesn't have a realistic tool to measure it, rather depends on exploring the populace perception on the subject then we say relative or subjective or qualitative ontology is 
adopted. ${ }^{19,20}$ Thus we have two types of ontological principle namely realism and relativism.

\section{Realistic Ontology}

Here the researcher believes that what he or she is 'trying to search' can be 'quantified and measured in absolute terms' like numbers and units.

\section{Relative Ontology}

In this approach, the researcher desires to know what people think about the subject that the researcher is trying to study. So the population perspective rather than the researcher view is the deciding point.

\section{Epistemology}

This is the second step in the research paradigm where we decide how to study the existing knowledge. Adoption of a realistic ontology will require an ETIC or analytical epistemological approach; whereas adopting a relative ontology will lead to EMIC or exploratory epistemology. ${ }^{21-25}$

\section{ETIC Epistemology}

ETIC means the researcher or observer observes the phenomenon from outside without being a part of it or party to it. ${ }^{11}$ Here all the measurements are recorded from the study population or participants by the researcher and his team members in a predefined manner; like using the well established measurable guideline. Examples are blood tests, different questionnaires etc. these measurable items can later be analysed by robust statistical tools and interpreted in a definitive manner so as to predict an outcome or accept or defuse a hypothesis. Hence it is researcher driven, exploring the views and concerns of the researcher.

Thus ETIC epistemology requires more of 'T's like statistical testing and uses a methodology with more 'T's i.e. quantitative rather than qualitative methodology.

\section{EMIC Epistemology}

In EMIC concept the researcher becomes a part of the study or a party to it. ${ }^{17}$ The $\mathrm{M}$ in EMIC stands for me i.e. insiders perspective. Say for example to study the behaviour or perception of the population on a particular subject he himself become a part of the process, mingles with them, conducts participants observation, in-depth interview and focused group discussions so as to come to a logical conclusion on what they believe about certain things like the 'researching question' of the researcher and why them think so. Here he or she tries to describe something from their (participants) perspective and uses "In vivo" quotes. It is actor-driven thereby expressing their views and concerns.

The M in EMIC stands for me i.e. insiders perspective. The ' $T$ 's are missing. Less of statistical testing and the methodology have less 'T's as well i.e. qualitative.

\begin{tabular}{|c|c|}
\hline \multicolumn{2}{|c|}{ ETIC Vs. EMIC epistemology } \\
\hline ETIC & EMIC \\
\hline Outsider view & Insider view \\
\hline In vitro & In vivo \\
\hline Analytical, statistic-based & Logical, inference based \\
\hline Quantitative approach & Qualitative approach \\
\hline Tests Hypothesis & $\begin{array}{c}\text { Generates a tentative } \\
\text { hypothesis }\end{array}$ \\
\hline $\begin{array}{c}\text { Meaningful to the } \\
\text { researcher }\end{array}$ & Meaningful to the actor \\
\hline
\end{tabular}

\section{Methodology}

This provides the researcher with different choices from which one can choose a correct and scientifically viable one. Broadly we have 3 types of research methodology i.e. quantitative, qualitative and mixed methods. ${ }^{26-28}$

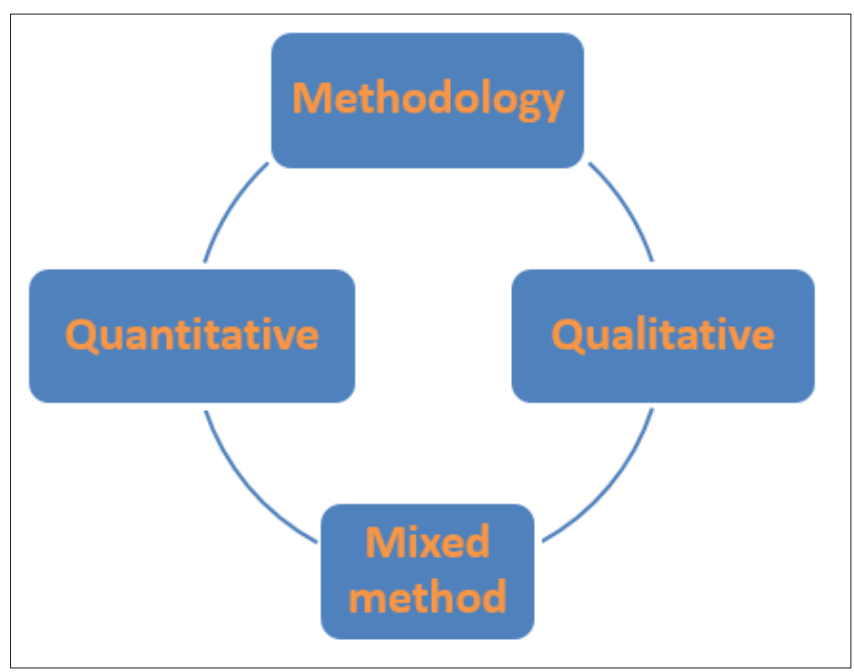

\section{Quantitative Methodology}

When a researcher has a hypothesis at hand and a tool through which it can be evaluated then the methodology that has to be adopted should be a quantitative one. The quantitative cafeteria provides one with options of observational or experimental research methods. The observational methods are cross-sectional studies, casecontrol studies or cohort studies to name the important ones. Whereas the experimental design provides options through different types of control trials including cross over designs. Once again the RQ decides which design to choose from.

\section{Qualitative Methodology}

One explores qualitative methodology so as to gain some firsthand information relating to a specific topic. If research is at an initial stage of hypothesis building and desires to know the population perspective of the problem then he 
toes the line of qualitative methodology. Here he has to decide from methods like Participant observation, in-depth interviews, and focus groups. Again his research method will be decided by what he is planning to explore or his RQ?

\section{Mixed Methodology (Mixed-Method Research)}

Gone are those days when exponents of quantitative and qualitative methodology were head-on heals trying to score over each other. Time has come to amend peace and have peaceful, productive and collaborative coexistence.

There is nothing which is absolute; this too includes 'knowledge' or 'truth'. Now it is widely accepted that what is true to-day may not be so tomorrow, or seeking an answer to a particular problem we may need to apply so-called diverging approaches or methods. In the field of research many times we are motivated to adopt both quantitative and qualitative approach to seek evidence and prove or disprove them. Thus in mixed-method researches both quantitative and qualitative methods lend support to each other so as to prove and propagate a concept. ${ }^{28,29}$

The methodology provides us with different available options or methods which can be adapted to carry out research.

\section{Methods}

The fourth and final step in the research paradigm if the research method. This means the actual procedure that is adopted to study or test the proposed concept. Here the details of the study protocol are spelt out. The study method, setting, participants, tools, outcome variables etc. are chalked out in detail so as to make the study robust, unbiased and interpretive. The examples are survey, CCS, CS and control trials for quantitative research and case history, in-depth interview, FGD for qualitative research.

The research method is the grass root level activities and planning in the research paradigm. Here the details of the process are chalked out and meticulously executed. One's ontological believe and epistemological approach will decide what methodology and method, in particular, shall be adopted.

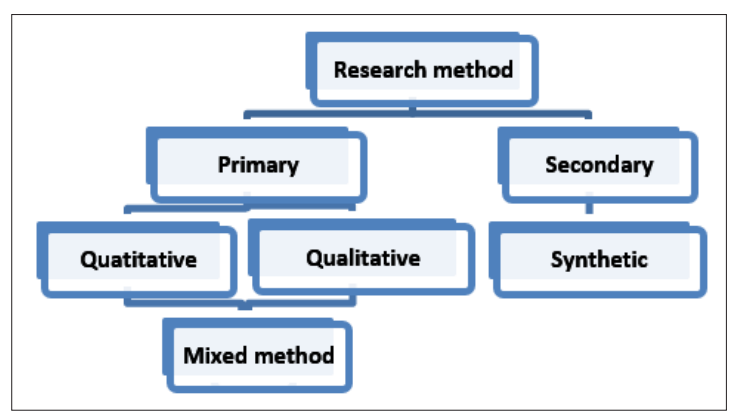

Research methods can be of two types i.e. primary and secondary. in primary, the researcher collects information prima facia, analyse and interpret them whereas in secondary research the researcher compiles and analyse related primary research data and observations so as to produce synthetic 'evidence-based guideline' for general use.

\section{Primary Research Method}

This is the most common and popular research method that is conducted and encountered on a routine basis. Here the researcher and his team finalize the protocol and act upon it. This involves finalizing issues like the population size(sample size), the parameters to be measured, who is to be and not be in the study(exclusion and inclusion criteria), what factors to be controlled(minimizing bias and confounding) etc. Depending on the researcher's primary belief we can either adopt a quantitative method or a qualitative one.

\section{Quantitative Methods}

This is the ideal method to supplant realistic ontology and ETIC epistemology. The research question determines which method to be employed. if we are interested in estimating the burden of a disease or a health-related condition then we should adopt a descriptive method which can find out the answer to 'who', 'where' and 'how' of the condition. the likely method to adopt is case studies, case series, survey or cross-sectional study design.

On the other hand, if we plan to explore associations and causality then an analytical observational study design of the nature of case-control or cohort would be the apt ones.

A desire to study outcomes of medicine and effects of investigations would need an 'analytical interventional' method like 'randomized control trial'.

Thus quantitative methods can be broadly classified into descriptive and analytical methods and the analytical one to further observational and international ones.

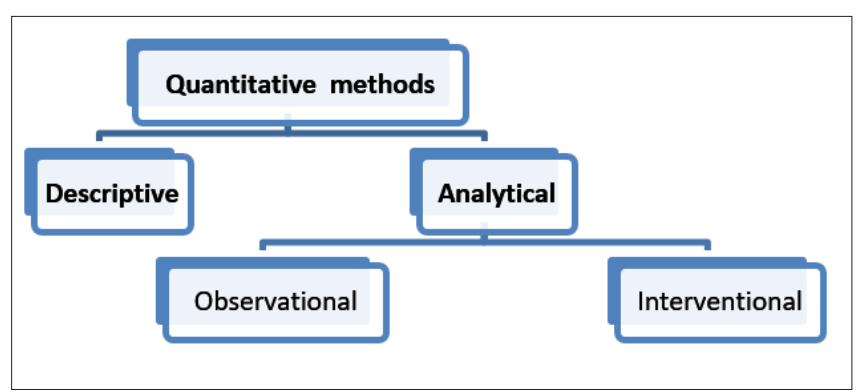

\section{Qualitative Methods}

Qualitative methods are adopted when we want to know the population perspectives on an issue. Here, instead of a predesigned and fixed set approach (quantitative approach); we employ an open mindset and record what the populace believes on the issues. Their opinion is recorded by different 
qualitative methods like participant observation, in-depth interviews and focus groups.

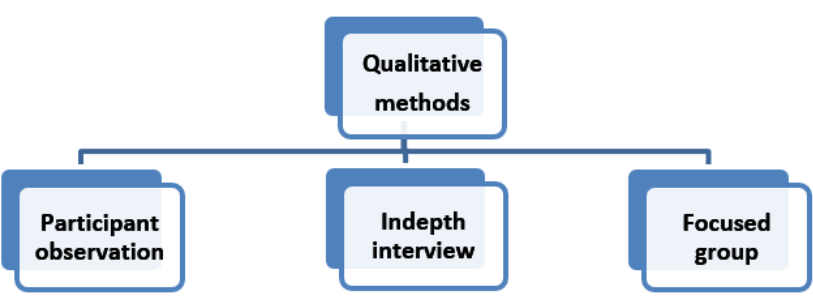

\section{Mixed Method Research}

Debates, discussions and differences have huge power in shaping up things. Research in general and in medicine, in particular, is no exception. It has experienced hot debates in the 1980s and 1990s, where protagonists were headon heals in dividing it into two contrasting categories. In one such differentiating attempt the group supporting quantitative approach were coined as 'scientists' and the other favouring qualitative methods were levelled 'detectives'. ${ }^{28-30}$

But over the years the bitterness has dampened. Now, these are no more considered as competitors but as collaborators. So we have 'collaborative' or 'mixed method' research; which is a welcome move.

This engaging work has given a new dimension to researching fraternity. In the modern era many a time an answer to a quantitative query is sought through qualitative approach and vice versa.

\section{Secondary/ Synthetic Methods}

The fruit of primary research is mostly raw in nature. In order to make it palatable, we need a ripening agent. The repining agent for quantitative research which aims to 'bring them closer to decision-making' is 'systematic review' and for qualitative research is 'thematic review'. ${ }^{31,32}$ In both these circumstances, their respective primary research methods are scrutinized on common lines and a feasible synthetic guideline is generated for popular use.

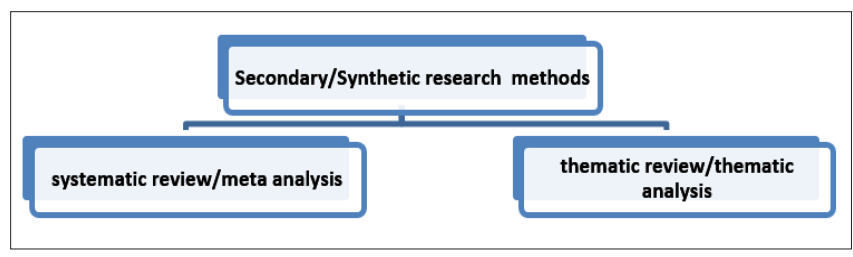

\section{Scenario (example) I}

To make things simple let us look into an example of violence among children and television watching. As an initial approach, we have to understand why children have violent behaviour. Thus in order to have firsthand information we should construct an exploratory research ontology which will allow us to generate a tentative hypothesis. Via exploratory ontology we plan to know about the existing believe in the populace about childhood violent behaviour and by EMIC epistemology we can gather corroborative information that points to available knowledge in this direction. Then we can test the knowledge so generated by applying qualitative methodology and specific qualitative methods like case studies, focused group discussions etc. All these may lead us to a tentative hypothesis that 'there is a popular belief that consistent exposure of children to television programs and video games is the cause for increased violent behaviour; as their thematic concept is perceived to propagate aggression and violence'.

Further, this tentative hypothesis can be tested through realistic ontology, ETIC epistemology, quantitative methodology and quantitative methods.

\section{Scenario (example) 2}

Let us see how all these are done. A befitting example can be the role of a dental care package in reducing blood sugar level and helping in long term control of T2DM (type 2 diabetes mellitus)'.

Let us explore this from differing research paradigms or principles. Let us consider that there is some truth in oral health-T2DM research statement. If we believe that this propagated concept of truth is objective and measurable then we are propagating realistic ontology as the first step in the research paradigm. On the contrary, if we are not sure of these but want to get an insight or firsthand information on this concept, then we have to rely on population perception on this subject. We will be interested in knowing what people think about this concept. Under this scenario, we shall be adopting a subjective or relativistic (logical) ontology.

As the second step in the research paradigm depending on our ontological believe we design or choose the epistemological concept. If we believe that we have the expertise or knowledge through which the impact of oral health care package on improving T2DM can be effectively measured then we are said to be pursuing ETIC epistemology. But in contrast, if our concept is naive then we can adopt EMIC epistemological principles where the existing population perception depending on their experiences can be evaluated and a tentative hypothesis generated for further exploration, preferably by realistic, measurable and accountable manner as is the case with realistic ontology and its subsequent research sequences.

The third step of the research paradigm looks after the available options through which the proposed concept can be studied and evaluated. these options constitute the methodological segment of the research paradigm. Here a realistic approach with ETIC epistemology can be followed upon by quantitative methodology whereas a relative or 
subjective approach with EMIC concept is devoured by qualitative methodology.

Under the quantitative approach, one can conduct a 'survey' or 'cross-sectional observation (study)' to find out how many of the T2DM patients are on the habit of getting regular dental checks and attentions and compare their blood sugar profile with the other section of the surveyed population who are not doing so. Or one can conduct a 'case-control study' by getting the data from T2DM cases and looking into or asking them about their dental visits and cares in past. From this information, two groups can be created one receiving regular dental care as cases and the other not doing so as to controls. The results of these two groups then can be compared. Furthermore, we can follow a group of T2DM cases over time as a cohort and divide them into two groups depending on their dental care. We may find that some of them may be getting regular dental attention in comparison to others. Thus we can compare the cohorts and find any association of regular dental attention with improved glycemic control. Even further we may adopt a Randomized Control Trial (RCT) protocol and divide matched T2DM population into intervention and control arm and administer a predesigned dental care package to the intervention group and a placebo package to control group. The data so collected can be analysed to see if the dental care group has a better disease with respect to the control one.

Thus we have different quantitative options (methods) in terms of the survey, case-control study, cohort observations (study) and control trials to propagate and evaluate the realistic and ETIC concepts.

Similarly, a relative or subjective concept can be studied by a qualitative methodology which includes case study, in-depth interview FGD where the researcher makes an attempt to study the population perspective on the subject of interest. Here the researcher collects information from the population by open-ended questions, read their transcripts and analyse voice records, decode them and make a logical assumption about the topic in questions. For example, the researcher can ask people to talk about their T2DM management, what they believe are contributing to better control and find out whether they believe/consider better oral care as one among them.

\section{Summary}

The best way we can explain these entangling concepts and terminologies is to find an appropriate metaphor. The research process can best be explained by imagining and comparing it with an iceberg. The visible tip of the iceberg represents only 10-12 per cent of the entire structure. Similarly what is reported in journals and works of literature are the research methods, thereupon representing the 'visible tip' of the research process. Further, this research method can be quantitative or qualitative depending on our research paradigm.

Like the massive structure that lies below the water surface which makes the tip visible and keeps the structure floating we have the research skeleton which constitutes of research methodology, epistemology, and ontological framework. We can divide this skeleton into two halves depending on what we plan to explore.

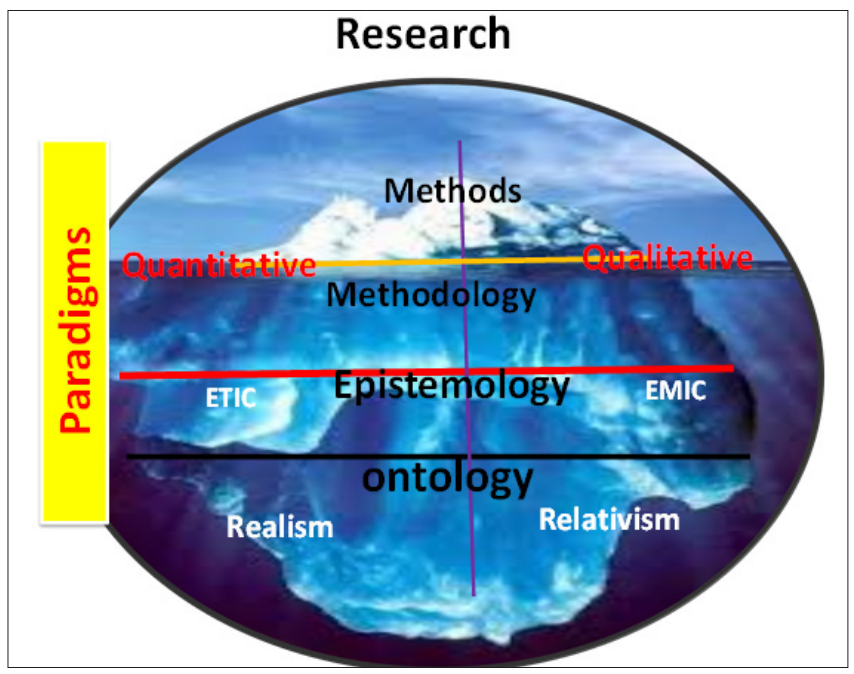

When we plan to explore certain items for the first time or on which much is not known then we restore to a subjective or explorative approach. This will lead us to know what are the common societal perception on the subject and the existing knowledge on them. From this, we may reach a tentative conclusion which is technically known as generating 'tentative hypotheses'. This half of the research paradigm is said to have a relative ontology, EMIC epistemology, qualitative methodology and qualitative methods.

On the other hand when we want to prove or disprove an item or concept or evidence, then we should adopt a realistic measurable approach which is objective in nature. Here we need to apply different statistical tests which will help us in making affirmative decisions in these regards. This type of research paradigm is said to have an objective ontological concept, ETIC epistemological guidelines and quantitative methodology and methods.

A research paradigm is the 'path' adopted to conduct research. Believing in truth is ontology, existing knowledge of truth is epistemology, the available ways to explore knowledge on truth is the methodology and the exact procedure adopted to explore and interpret knowledge is the method.

\section{Conflict of Interest: None}




\section{References}

1. The Editors of Encyclopaedia Britannica.Philosophy; https://www.britannica.com/topic/philosophy; December 14, 2018. Access on March 09, 2020.

2. Pranas Žukauskas, Jolita Vveinhardt and Regina Andriukaitiené (April 18th 2018). Philosophy and Paradigm of Scientific Research, Management Culture and Corporate Social Responsibility, Pranas ?ukauskas, Jolita Vveinhardt and Regina Andriukaitien?, Intech Open, DOI: 10.5772/intechopen.70628.

3. Saunders M, Lewis $P$, Thornhill A. Research Methods for Business Students" $6^{\text {th }}$ edition, Pearson Education Limited. 2012.

4. Blaikie, N. Designing Social Research. Polity Press. 2010.

5. Creswell JW. Research Design: Qualitative and Quantitative and MixedMethod Approaches, Thousand Oaks, CA: Sage Publications. 2003.

6. Punch K. Introduction to Social Research: Quantitative and qualitative approaches, London: Sage Publications. 2005.

7. Hughes J. The Philosophy of Social Research, $3^{\text {rd }}$ Ed., London: Longman. 1997.

8. Moon K, Blackman D. A Guide to Understanding Social Science Research for Natural Scientists. Conservation Biology 2014; 28: 1167-1177.

9. Egon G Gura. Relativism, Curriculum Inquiry, 1992; 22(1): 17-23. DOI: 10.1080/03626784.1992. 11075390.

10. Caracelli VJ, Greene JC. Data Analysis Strategies for Mixed-Method Evaluation Designs. Educational Evaluation and Policy Analysis 1993; 15(2): 195207. https://doi.org/10.3102/01623737015002195.

11. Keser K, Köksal K, Keystones of research: epistemological and ontological analysis of educational studies. International Association of Research in Foreign Language Education and Applied Linguistics ELT Research Journal 2017; 6(4): 294-301.

12. Guba EG, Lincoln YS. Competing paradigms in qualitative research. In: Denzin NK, Lincoln YS, editors. Handbook of Qualitative Research. Thousand Oaks, CA: Sage; 1994; 105-117.

13. Gliner JA, Morgan GA. Research Methods in Applied Settings: An Integrated Approach to Design and Analysis. New Jersey, London: Lawrence Erlbaum Associates; 2000.

14. Mackenzie N, Knipe S. Research dilemmas: Paradigms, methods and methodology. Issues in Educational Research 2006; 16(2): 1-11.

15. Bounds G, Yorks L, Adams M, Ranney G. Beyond Total Quality Management: Toward the Emerging Paradigm. New York: McGraw-Hill; 1994; 832.

16. Olsen W, Morgan J. A Critical Epistemology of Analytical Statistics: Addressing the Sceptical Realist. Journal for the Theory of Social Behaviour 35(3): 0021-8308.

17. Long RG, White MC. William H. Friedman \& Deborah V. Brazeal. The 'Qualitative' Versus 'Quantitative' Research Debate: A Question of Metaphorical Assumptions? International Journal of Value-Based Management 2000: 13; 189-197.

18. John Mingers, Alistair Mutch and Leslie Willcocks. Critical Realism in Information Systems Research 2013; 37(3): 795-802.

19. Healy M, Perry C. Comprehensive criteria to judge validity and reliability of qualitative research within the realism paradigm. Qualitative Market Research 2000; 3(3): 118126. https://doi.org/10.1108/13522750010333861.

20. Gary A, Carlos RA, Vivas LS, Palacios MD. Ontological supports of knowledge: knowledge creation and analytical knowledge. Management Decision 2011; 49(2): 183-194. https://doi.org/ 10.1108/00251741111109106.

21. Bryman A. Social Research Methods : New York: Oxford University Press. 2001.

22. Denzin NK, Lincoln Y. The Landscape of Qualitative Research: Theories and Issues. Thousand Oaks, California: Sage. 1998.

23. Dilts R, Delozier J. Encyclopedia of NLP: Santa Cruz: NLP University Press. 2000.

24. Atkins S, Murphy K. Reflection: A review of the literature. Journal of Advanced Nursing 1993; 18(8): 1188-1192.

25. Barnstone A, Barnstone W. A book of women poets from antiquity to now. New York: Schocken Books. 1980.

26. Walliman NS, Walliman N. Research methods: the basics" Taylor and Francis. 2011.

27. Brown RB. Doing Your Dissertation in Business and Management: The Reality of Research and Writing, Sage Publications. 2006.

28. Allan AJ, Randy LJ. Writing the Winning Thesis or Dissertation. A Step-by-Step Guide, Corwin Press, California. 2005.

29. Shorten A, Smith J. Mixed methods research: expanding the evidence base. Evidence-Based Nursing 2017; 20: 74-75.

30. Aramo-Immonen H. Mixed Methods Research Design. In: Lytras M.D., Ruan D., Tennyson R.D., Ordonez De Pablos P., García Peñalvo F.J., Rusu L. (eds) Information Systems, E-learning, and Knowledge Management Research. WSKS 2011. Communications in Computer and Information Science, vol 278. Springer, Berlin, Heidelberg. 2013. https://doi.org/10.1007/978-3642-35879-1_5.

31. Castleberry A, Nolen A. Thematic analysis of qualitative research data: Is it as easy as it sounds. Currents in Pharmacy Teaching and Learning 2018; 10(6): 807-815.

32. Haidich AB. Meta-analysis in medical research. Hippokratia 2010; 14(Suppl 1): 29-37. 